\title{
Uso de rastreadores para busca de reações adversas a medicamentos como motivo de admissão de idosos em pronto-socorro
}

\author{
Use of triggers tools to search for adverse drug reactions \\ in the elderly admitted to emergency departments
}

Kelly Lie Nagai ${ }^{1}$

Patricia Sayuri Katayose Takahashi ${ }^{1}$

Lucia Mendes de Oliveira Pinto ${ }^{1}$

Nicolina Silvana Romano-Lieber ${ }^{2}$

${ }^{1}$ Hospital Universitário de São Paulo, Universidade de São Paulo (USP). Av. Prof. Lineu Prestes 2565, Butantã. 05508-000 São Paulo SP

Brasil. kellylie17@gmail.com

${ }^{2}$ Faculdade de Saúde

Pública, USP. São Paulo

SP Brasil.

\begin{abstract}
Adverse drug reactions (ADRs) can cause illness, disability or death, especially in the elderly. An active search for suspected ADRs was carried out using triggers, which motivated the search of elderly people under care in adult emergency departments (ED). It was a cross-sectional and retrospective study that used an adaptation of the Institute of Health Care Improvement triggers. A total of 287 medical records were analyzed and 38 triggers were found, identifying 7 suspected ADRs. One was found without the use of triggers. Thus, in total, 8 ADRs (2.79\%) were found, of which 6 were considered serious. There was a higher prevalence of ADRs in females (62.5\%) and in those over 80 years of age (50\%). The medications most implicated were those for alimentary tract and metabolism and cardiovascular system. Of the triggers tested, some are essential for use at EDs, such as those that indicate problems with anticoagulants, hypoglycemic agents and antihypertensives. Triggers have proved useful for an active search for suspected ADRs at EDs, including severe ones, identifying problems occurring outside hospital settings and signaling medications that pose an increased risk to the elderly. Key words Drug-related side effects and adverse reactions, Pharmaceutical preparations, Emergency medical services, Aged
\end{abstract}

Resumo Reações adversas a medicamentos (RAM) podem ser causa de enfermidades, incapacidades ou óbitos, principalmente em idosos. Realizou-se busca ativa de suspeitas de RAM que motivaram a procura de idosos por cuidados em pronto socorro (PS) utilizando rastreadores e discutindo seu uso nessa unidade de atendimento. Tratou-se de um estudo transversal e retrospectivo que utilizou adaptação dos rastreadores do "Institute of Healthcare Improvement". Foram analisados 287 prontuários, nos quais foram encontrados 38 rastreadores que identificaram 7 suspeitas de RAM. Uma foi encontrada sem uso dos rastreadores. Assim, no total, foram encontradas 8 reações (2,79\%). Os medicamentos mais implicados foram os para trato alimentar e metabolismo $e$ sistema cardiovascular. Dos rastreadores testados, alguns são essenciais para uso em PS, como os que indicam problemas com anticoagulantes, hipoglicemiantes e anti-hipertensivos, medicamentos bastante utilizados por idosos. Outros são adequados a estudos prospectivos, por necessitarem avaliação detalhada para confirmar da suspeita de RAM. Os rastreadores devem ser adaptados para cada instituição, mas se mostraram úteis para a detecção das suspeitas de RAM, identificando problemas ocorridos fora do ambiente hospitalar e sinalizando medicamentos que envolvem maior risco ao paciente idoso.

Palavras-chave Reação adversa, Medicamento, Pronto-socorro, Idoso 


\section{Introdução}

Medicamentos são utilizados para trazer benefícios a quem os utiliza, entretanto, algumas reações adversas a medicamentos (RAM) podem causar enfermidades, incapacidades ou óbitos ${ }^{1}$ Além disso, quando ocorrem em pacientes internados, aumentam o tempo de internação, interrompem tratamentos e aumentam as despesas hospitalares ${ }^{2}$. Revisão recente, realizada com estudos europeus, revelou que $10,1 \%$ dos pacientes experimentaram uma RAM durante a hospitalização ${ }^{3}$. Esse valor chegou a 16,8\% em meta-análise que estimou o surgimento de RAM no decorrer da internação $0^{4}$. Além disso, outra meta-análise apontou que as RAM estão entre a $4^{\text {a }}$ e a $6^{\text {a }}$ causa de morte nos Estados Unidos, na população em geral, com uma incidência estimada de 0,23 a $0,41 \% \%^{5}$.A prevalência de RAM pode variar devido à heterogeneidade da população estudada, a metodologia utilizada para sua detecção e o tipo de instituição envolvida ${ }^{4}$.

Reações adversas a medicamentos têm sido apontadas como associadas à admissão hospitalar entre $0,16 \%$ a $15,7 \%$, dependendo da idade dos pacientes ${ }^{6}$. Meta-análise demonstrou que a chance de internação devido à suspeita de RAM em pacientes idosos é quatro vezes maior do que em pacientes não-idosos ${ }^{7}$. Isso pode ser explicado pelas alterações fisiológicas que ocorrem no organismo provocadas pelo envelhecimento, como na farmacocinética e na farmacodinâmica, o que influencia a metabolização e eliminação dos medicamentos pelo organismo, fazendo com que os idosos sejam mais susceptíveis a $\mathrm{RAM}^{8}$, mas também pode ocorrer porque pacientes idosos, em geral, recebem múltiplos medicamentos para doenças crônicas.

A detecção de RAM no Pronto Socorro Adulto (PSA) é importante porque alguns estudos mostram que uma significativa taxa de admissão ocorre devido a medicamentos utilizados no domicílio do paciente ${ }^{9,10}$. Entre os fatores para a essa ocorrência pode-se citar a automedicação, já que segundo meta-análise realizada no país, um terço da população brasileira adulta (18 a 65 anos) se automedica ${ }^{11}$. Além disso, a sobreutilização de medicamentos e o seu uso irracional aumentam a probabilidade de ocorrência de um evento, resultando em gasto desnecessário e evitável dos recursos públicos para a saúde ${ }^{8}$.

Para a busca desses eventos em pacientes hospitalizados tem sido utilizada uma estratégia denominada rastreador. Um rastreador pode ser encontrado a partir da revisão do prontuário do paciente e sua presença permite direcionar a investigação para determinar a ocorrência e a mensuração do evento. Os rastreadores podem ser (a) medicamentos prescritos para tratar um possível evento, (b) resultados de exames laboratoriais e (c) procedimentos e intervenções da equipe de saúde ou mesmo a suspensão abrupta de um medicamento ${ }^{12}$.

O objetivo deste trabalho foi realizar a busca de suspeitas de RAM que motivaram a procura de idosos por cuidados no PSA de um hospital universitário de média complexidade, utilizando rastreadores e discutir seu uso nessa unidade de atendimento.

\section{Métodos}

Trata-se de um estudo transversal retrospectivo realizado no PSA de um hospital universitário de média complexidade, localizado no Município de São Paulo.

Foram incluídos no estudo, pacientes com idade igual ou superior a 60 anos que deram entrada no PSA durante os períodos definidos para a pesquisa. Para se obter uma amostra mais heterogênea de atendimento de um PSA, foram escolhidos os meados de cada estação do ano, para evitar o viés de sazonalidade. Assim, a coleta de dados foi baseada num intervalo de 28 dias divididos ao longo do ano em 4 períodos: de 20 a 26 de julho 2013, de 20 a 26 de outubro 2013, de 20 a 26 de janeiro de 2014 e de 20 a 26 de abril de 2014.

Foram excluídos do estudo os pacientes cujo prontuário ou ficha de atendimento no PSA não estavam disponíveis no Serviço de Arquivo Médico e Estatística (SAME).

O Setor de Informática do hospital disponibilizou a lista dos pacientes atendidos no PSA, no período definido para o estudo. Utilizando-se os critérios de inclusão e exclusão, os pacientes foram selecionados randomicamente a partir dessa lista. Os prontuários foram solicitados ao SAME para a busca dos rastreadores. Foi feita leitura da evolução clínica, das prescrições e das anotações da equipe multidisciplinar (enfermagem, fisioterapia, terapia ocupacional e fonoaudiologia). Já os resultados de exames e os respectivos valores de referência utilizados no hospital, foram consultados no sistema eletrônico interno da instituição.

Os rastreadores foram baseados no estudo do Institute for Healthcare Improvement (IHI) ${ }^{13}$, o qual desenvolveu uma lista de rastreadores globais que permite mensurar os eventos adversos e 
que podem ser utilizados em qualquer serviço de saúde. Não eram específicos para PSA, mas nesse estudo foi analisada sua aplicabilidade nesse local, já que são os mais frequentemente citados na literatura e de fácil utilização.

Para este trabalho foram pesquisadas apenas suspeitas de RAM, as quais são definidas como qualquer evento nocivo e não intencional causado por medicamentos em doses usualmente utilizadas com a finalidade profilática, terapêutica ou diagnóstica ${ }^{14}$. Trata-se de um tipo particular de evento adverso a medicamento (EAM), já que o EAM é um termo mais amplo, definido como qualquer ocorrência médica desfavorável que pode aparecer durante o tratamento medicamentoso, mas que não apresenta necessariamente uma relação causal com esse tratamento ${ }^{14}$. Dessa forma, não foram pesquisados erros de medicação, intoxicações, uso off-label e queixas técnicas.

Como foram abordadas as suspeitas de RAM, e não a totalidade de eventos adversos, como o IHI propõe, foram utilizados apenas os rastreadores apresentados no Módulo de Medicamen$\operatorname{tos}^{13}$. A lista do IHI foi adaptada, já que para os exames laboratoriais foi utilizado o valor de referência adotado pelo Laboratório Clínico do hospital estudado. A lista de rastreadores é apresentada no Quadro 1. Com os resultados obtidos, foi realizada uma análise descritiva por meio de frequências relativas sobre o paciente com a suspeita de RAM, a caracterização da suspeita de RAM e os medicamentos envolvidos.

Para a caracterização dos medicamentos envolvidos, utilizou-se o $1^{\circ}$ e o $5^{\circ}$ níveis da Classificação Anatomical Therapeutic Chemical (ATC) ${ }^{15}$. Foram também registrados a posologia utilizada e o time-window, o qual verifica a coerência entre o tempo da administração dos medicamentos e o do início da RAM.

As suspeitas de RAM foram classificadas segundo o World Health Organization - Adverse Drug Reaction Terminology (WHO-ART), que apresenta níveis de classificação. Foi utilizado o nível SOC (Sistema, Órgão, Classe $)^{16}$. Assim, puderam ser analisados quais sistemas e órgãos foram atingidos pelas suspeitas de RAM.

A gravidade da RAM foi definida pelo critério contido nas "Diretrizes para o Gerenciamento do Risco em Farmacovigilância” da Agência Nacional de Vigilância Sanitária (Anvisa), segundo as quais a RAM é considerada grave quando se verificam: óbito, ameaça à vida, hospitalização ou prolongamento da internação, incapacidade persistente ou significativa, anomalia congênita e evento clinicamente significativo (necessidade da intervenção médica a fim de se evitar o óbito, risco à vida ou atendimento hospitalar $)^{17}$.

Já a análise de causalidade foi realizada utilizando-se o algoritmo de Naranjo, o qual avalia a probabilidade de que uma suspeita de RAM seja consequência do uso do medicamento. As categorias de causalidade são: definida, provável, possível e duvidoso ${ }^{18}$.

O desfecho da RAM referiu-se à evolução do paciente em relação a suspeita de RAM apresentada: foi tratado e recebeu alta, houve necessidade de internação, evoluiu para óbito ou evadiu do hospital. Todas as suspeitas de RAM encontradas nesse trabalho foram discutidas com o grupo de Farmacovigilância do hospital pesquisado, para uma análise mais acurada do caso e a sua notificação para a Anvisa, se indicado.

O trabalho foi aprovado pelos Comitês de Ética das instituições envolvidas.

\section{Resultados}

No período de 28 dias estudados foram atendidos 2.326 pacientes com idade igual ou superior a 60 anos, no PSA do hospital. Desses pacientes, foram excluídos 828 idosos que apresentavam apenas uma ficha de atendimento no arquivo. Dos 1.498 restantes, foram solicitados ao SAME 475 prontuários, de forma aleatória. Não foi possível encontrar a ficha de atendimento em todos os prontuários, pois alguns haviam se perdido e outros não existiam devido à evasão de pacientes após a abertura da ficha de atendimento e antes do atendimento do profissional de saúde. A Figura 1 ilustra o resultado do estudo.

No total, foram observadas 8 suspeitas de RAM como motivo de entrada no PSA após a análise de 287 prontuários, o que equivale a uma prevalência de 2,79\% de RAM. No Quadro 2 apresenta-se as suspeitas de RAM encontradas.

O perfil demográfico dos pacientes que apresentaram suspeitas de RAM está apresentado na Tabela 1.

As suspeitas de RAM encontradas, de acordo com o WHO-ART, foram classificadas como 0800 (desordens metabólicas e nutricionais), 0410 (desordens do sistema nervoso central e periférico), 0600 (desordens do sistema gastrointestinal) e 0431 (desordens visuais). Todas as suspeitas de RAM foram tratadas no PSA e os pacientes tiveram alta no mesmo dia. Apenas a constipação e a diplopia foram consideradas como não-graves. Em todos os casos, o time-window entre a suspeita de RAM e a utilização do medicamento 
Quadro 1. Lista de rastreadores* utilizados para busca de suspeitas de Reações Adversas a Medicamentos levando idosos a atendimento em Pronto Socorro - São Paulo - 2013-2014.

\begin{tabular}{|c|c|}
\hline Rastreador & Significado \\
\hline $\begin{array}{l}\text { M1. Cultura de fezes positiva para } \\
\text { Clostridium difficile }\end{array}$ & Rastreador para reação adversa a antimicrobianos. \\
\hline $\begin{array}{l}\text { M2. Tempo de tromboplastina } \\
\text { parcial (TTP) maior que } 100 \\
\text { segundos }\end{array}$ & $\begin{array}{l}\text { Valores aumentados ocorrem em uso de heparina. Para caracterizar } \\
\text { a suspeita de RAM, é necessário verificar se houve sangramento, } \\
\text { hematomas ou redução nos valores de hemoglobina e/ou hematócrito, } \\
\text { além do valor do TTP. }\end{array}$ \\
\hline $\begin{array}{l}\text { M3. Índice Internacional } \\
\text { Normalizado (INR) maior que } 6\end{array}$ & $\begin{array}{l}\text { Verificar presença de sangramento junto com o INR alterado para } \\
\text { indicar uma suspeita de RAM. }\end{array}$ \\
\hline $\begin{array}{l}\text { M4. Níveis de glicose menores que } \\
50 \mathrm{mg} / \mathrm{dL}\end{array}$ & $\begin{array}{l}\text { Verificar se o paciente apresentou letargia ou tremores ou se foi } \\
\text { prescrita administração de glicose. A presença desses fatores pode ser } \\
\text { indicativa de RAM devido à utilização de insulinas ou hipoglicemiantes. }\end{array}$ \\
\hline $\begin{array}{l}\text { M5. Nitrogênio Uréico Sanguíneo } \\
\text { maior que } 49 \mathrm{mg} / \mathrm{dL} \text { ou creatinina } \\
\text { sérica maior que } 1,30 \text { em homens e } \\
1,10 \text { em mulheres }\end{array}$ & $\begin{array}{l}\text { Verificar histórico do paciente para descartar outras causas da alteração } \\
\text { renal como doença renal pré-existente ou diabetes. }\end{array}$ \\
\hline M6. Administração de vitamina $\mathrm{K}$ & $\begin{array}{l}\text { Se for administrada devido a INR prolongado, revisar prontuário para } \\
\text { verificar evidências de sangramento. }\end{array}$ \\
\hline $\begin{array}{l}\text { M7. Administração de anti- } \\
\text { histamínicos }\end{array}$ & $\begin{array}{l}\text { São frequentemente utilizados para reações alérgicas a medicamentos, } \\
\text { mas também para outros procedimentos, como adjuvante } \\
\text { medicamentoso ou para alergias sazonais. Revisar prontuários para } \\
\text { determinar motivo do uso. }\end{array}$ \\
\hline M8. Administração de flumazenil & Pode indicar hipersedação ou sobredose por benzodiazepínicos. \\
\hline M9. Administração de naloxona: & $\begin{array}{l}\text { Pode indicar hipersedação, depressão respiratória, sobredose ou rigidez } \\
\text { torácica por medicamentos derivados de opióides. }\end{array}$ \\
\hline M10. Administração anti-emético & $\begin{array}{l}\text { Náuseas e vômitos são comumente resultado da administração de } \\
\text { medicamentos. Verificar se são devidos ao medicamento ou à condição } \\
\text { do paciente. }\end{array}$ \\
\hline M11. Supersedação/hipotensão & $\begin{array}{l}\text { Verificar sinais de supersedação e letargia. Analisar anotações e gráficos } \\
\text { de sinais vitais para verificar sinais de hipotensão relacionados com a } \\
\text { administração de sedações, analgésicos ou relaxantes musculares. }\end{array}$ \\
\hline $\begin{array}{l}\text { M12. Interrupção abrupta de } \\
\text { medicamento }\end{array}$ & $\begin{array}{l}\text { Pode indicar o medicamento suspeito de causar o evento adverso. Não } \\
\text { deve ser considerado como rastreador quando a suspensão se deu por } \\
\text { término de tratamento. }\end{array}$ \\
\hline M13. Outros & $\begin{array}{l}\text { Usar quando uma RAM for detectada, mas não estiver relacionada a } \\
\text { nenhum dos rastreadores desta lista. }\end{array}$ \\
\hline
\end{tabular}

*Adaptado do Medication Module Trigger do Institute for Healthcare Improvement ${ }^{13}$.

estava de acordo com o descrito na literatura. Ao realizar a análise de causalidade por Naranjo, todas foram consideradas possíveis.

Nos prontuários só constavam a posologia da metformina e da glibenclamida, as quais estavam sendo utilizadas corretamente. Já dos outros medicamentos, não foi possível confirmar a posologia prescrita, devido à falta de informação nos prontuários.
No total, foram encontrados 38 rastreadores nos 287 casos estudados, que estão expostos na Tabela 2.

Não foram encontrados os rastreadores $\mathrm{M} 1$, M2, M3, M6, M8 e M9. Os rastreadores M5, M7 e M10, foram encontrados, porém não estavam relacionados a nenhuma suspeita de RAM. Apenas os rastreadores M4 e M11estavam relacionados a suspeita de RAM. Para M13, há a sugestão de 2 


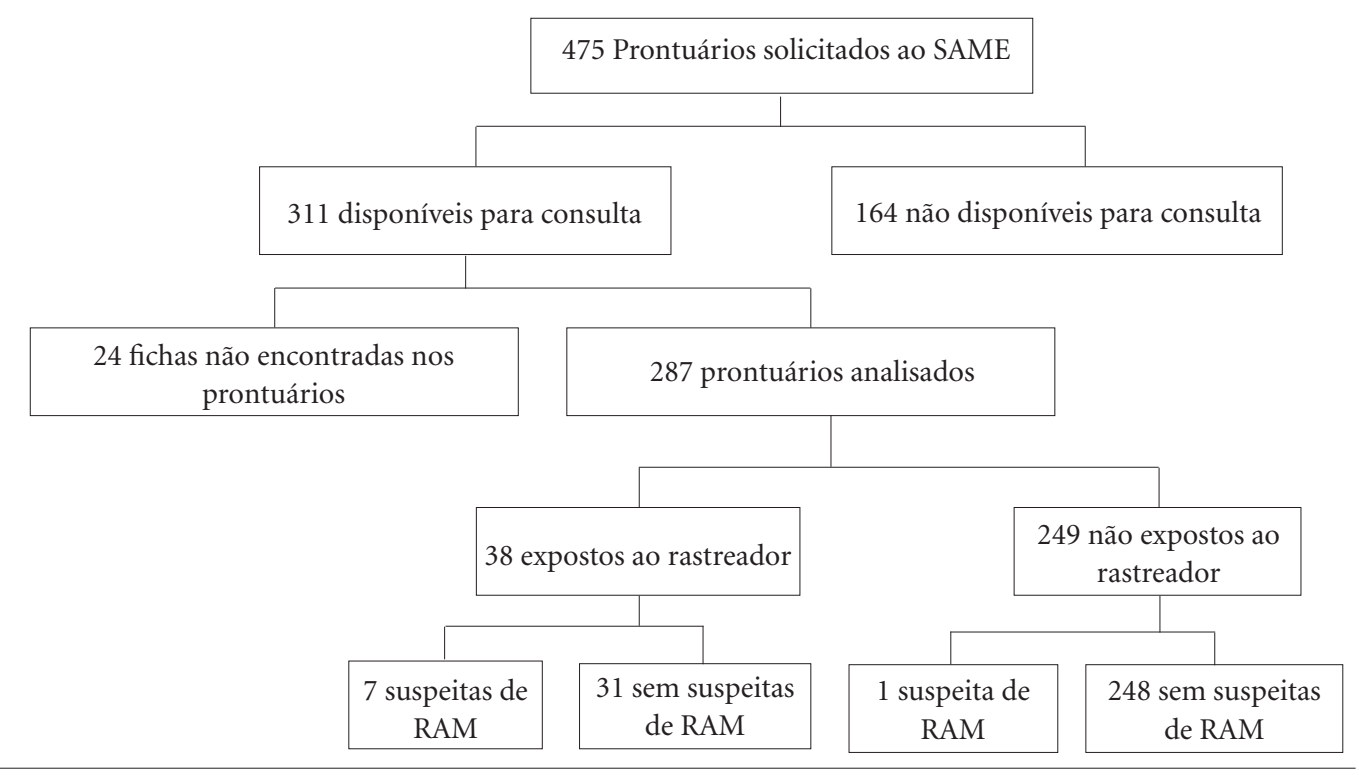

Figura 1. Distribuição dos prontuários e das suspeitas de RAM.

Quadro 2. Suspeitas de reações adversas a medicamentos que levaram pacientes idosos a atendimento em Pronto Socorro Adulto, com os rastreadores que os localizaram e os medicamentos suspeitos de causarem a reação- 2013 a 2014.

\begin{tabular}{|l|l|l|}
\hline \multicolumn{1}{|c|}{ Suspeitas de RAM } & \multicolumn{1}{|c|}{ Rastreadores } & \multicolumn{1}{c|}{ Medicamentos suspeitos (ATC)* } \\
\hline Hiponatremia & M13 (Outros): Nível sérico sódio $<136 \mathrm{mEq} / \mathrm{L}$ & Hidroclorotiazida(C) \\
\hline Hipoglicemia & M4 (Glicose $<100)$ & Glibenclamida (A) \\
\hline Hipoglicemia & M4 (Glicose $<100)$ & Metformina (A), Glibenclamida (A) \\
\hline Hipotensão postural & M11 (Hipotensão) & Captopril (C), Furosemida (C) \\
\hline Hipotensão postural & M11 (Hipotensão) & $\begin{array}{l}\text { Losartana (C), Hidralazina (C), } \\
\text { Amiodarona (C) }\end{array}$ \\
\hline Constipação & M13 (Outros): Constipação & Codeína (N) \\
\hline Constipação & M13 (Outros): Constipação & Poliestirenossulfonato de cálcio (V) \\
\hline Diplopia & - & Colírio de Timolol (S) \\
\hline
\end{tabular}

*os grupos entre parênteses representam o $1^{\circ}$ nível da Classificação Anatomical Therapeutic Chemical- ATC.

rastreadores: nível sérico de sódio e constipação. Uma suspeita de RAM (diplopia) foi encontrada sem a presença de nenhum rastreador. Diplopia não foi considerada como uma sugestão de rastreador útil para constar em M13, já que só foi possível constatar a suspeita de RAM, devido ao relato do médico no prontuário.

\section{Discussão}

Nesse estudo, verificou-se que 2,79\% dos casos de procura do PSA do HU-USP por idosos foram devidos a alguma suspeita de RAM. A literatura apresenta resultados bastante diversos.
Tabela 1. Características demográficas dos idosos que apresentaram suspeitas de reações adversas a medicamentos (RAM) que levando a atendimento em Pronto-Socorro - Município de São Paulo - 2013 - 2014.

\begin{tabular}{ccc}
\hline Características & $\begin{array}{c}\text { Prontuários } \\
\text { analisados }\end{array}$ & $\begin{array}{c}\text { Pacientes com } \\
\text { suspeita de RAM }\end{array}$ \\
\hline Sexo & & \\
Feminino & $167(58 \%)$ & $5(62,5 \%)$ \\
Masculino & $120(42 \%)$ & $3(37,5 \%)$ \\
Idade (anos) & & \\
$60-70$ & $126(44 \%)$ & $3(37,5 \%)$ \\
$71-80$ & $102(35 \%)$ & $1(12,5 \%)$ \\
$\geq 81$ & $59(21 \%)$ & $4(50,0 \%)$ \\
\hline
\end{tabular}


Tabela 2. Rastreadores presentes e rastreadores que indicaram suspeitas de reações adversas a medicamentos (RAM) em prontuários de idosos atendidos em Pronto Socorro - Município de São Paulo - 2013-2014.

\begin{tabular}{lcc}
\hline \multicolumn{1}{c}{ Rastreadores } & Presentes nos prontuários & Que indicaram RAM \\
\hline M1 (cultura de C.difficile positiva) & 0 & 0 \\
M2(TTP $>100)$ & 0 & 0 \\
M3 (INR $>6)$ & 0 & 0 \\
M4 (glicose $<100)$ & 2 & 2 \\
M5(uréia $>49$ e/ou creatinina alterada) & 21 & 0 \\
M6 (vitamina K) & 0 & 0 \\
M7 (anti-histamínico) & 1 & 0 \\
M8 (flumazenil) & 0 & 0 \\
M9 (naloxona) & 0 & 0 \\
M10 (anti-emético) & 9 & 0 \\
M11 (hipotensão/supersedação) & 2 & 2 \\
M12 (interrupção abrupta de medicamento) & 0 & 0 \\
M13 (outros) & 3 & 3 \\
Total & 38 & 7 \\
\hline
\end{tabular}

*Nível sérico de sódio alterado e constipação.

Revisão sistemática que analisou apenas estudos prospectivos observou que a prevalência de RAM em internações hospitalares variou entre 0,16\% a $15,7 \%$, com uma média de $5,3 \%$ para todas as idades, sendo $10,7 \%$ para idosos ${ }^{6}$. Estudo realizado em um hospital de nível terciário, em Barcelona, verificou que $3,3 \%$ dos idosos admitidos na urgência tinham uma suspeita de RAM como razão da admissão ${ }^{19}$.

Por outro lado, estudo realizado em Ontário, Canadá, avaliou o motivo de entrada no PSA de pacientes acima de 65 anos e verificou-se que $0,75 \%$ do total de atendimentos anuais foram devido a RAM e, dentre esses pacientes, $21,7 \%$ necessitaram de internação hospitalar. ${ }^{9}$ Outro estudo, também realizado no Canadá, com pacientes maiores que 65 anos, verificou que $0,8 \%$ dos motivos de atendimento no PSA eram devido a suspeitas de RAM, dos quais 21,5\% necessitaram de internação hospitalar ${ }^{10}$. Já nos Estados Unidos, foi encontrada uma taxa de $0,2 \%$ de pacientes maiores de 65 anos de idade que deram entrada no PSA devido a uma RAM (taxa mais de duas vezes maior do que em pacientes menores que 65 anos ${ }^{20}$. Os três últimos estudos se referiam a trabalho retrospectivo realizado em mais de um hospital. Os dois estudos canadenses se basearam no Código Internacional de Doenças (CID) que cada paciente apresentou como motivo de entrada no PSA e o estudo dos Estados Unidos se baseou no banco de dados nacional de eventos adversos. Todos eram estudos de busca passi$\mathrm{va}^{9,10,20}$. Isso pode ter influenciado nas diferenças de resultado apresentado, pois no atual trabalho foi realizada busca ativa em todos os prontuários, sem pesquisar o CID de entrada.

Nesses outros países a gravidade das RAM também era maior, já que dois deles mostraram uma taxa de hospitalização de aproximadamente $21 \%$ dentre os pacientes que apresentaram RAM como motivo de entrada no $\mathrm{PSA}^{9,10}$ e, neste trabalho, nenhum atendimento evoluiu para internação.

Um estudo brasileiro pesquisou problemas relacionados a medicamentos levando a atendimento em serviço de emergência. Nele, os autores verificaram que $9 \%$ dos problemas ocorreram em indivíduos com 65 anos ou mais, mas estes se referiam a indicação, eficácia e segurança, sendo que $29,2 \%$ eram suspeitas de RAM. Entretanto, não foram especificadas as faixas etárias desses pacientes, dificultando a comparação com os resultados deste estudo ${ }^{21}$.

As diferenças das prevalências encontradas nos diferentes trabalhos podem ter sido resultado das diferentes definições utilizadas para designar reação adversa, mas também podem ser devido à idade utilizada para se considerar alguém idoso, já que no Brasil são consideradas idosas pessoas com 60 anos ou mais enquanto em países desenvolvidos, idosos são os indivíduos com idade igual ou superior a 65 anos $^{22}$. Também o tipo de busca utilizada pode influenciar a prevalência, uma vez que estudos em que há revisão de prontuários combinada com entrevistas ao paciente reportam prevalências maiores ${ }^{6}$. Por outro lado, 
estudos retrospectivos apresentam taxas inferiores de prevalência ${ }^{23}$. O perfil do hospital em que o estudo foi realizado e até a duração do estudo também podem influenciar nos resultados. Neste último caso, há autores que afirmam que estudos mais longos apresentam prevalências menores que estudos mais curtos ${ }^{7}$.

Não surpreende que os medicamentos mais relacionados às suspeitas de RAM tenham sido os da classe A (Trato alimentar e metabolismo) e C (Sistema cardiovascular), já que segundo dados do Departamento de Informática do SUS (DataSUS), referentes ao ano de 2013, doenças crônicas como hipertensão, hipercolesterolemia e diabetes apresentam alta prevalência na população idosa brasileira, respectivamente, 50,6\%, 24,3\% e $18,1 \%{ }^{24}$. Também a revisão de Kongkaew e colaboradores evidenciou que medicamentos cardiovasculares estão entre os mais frequentemente associados à admissão hospitalar de idosos ${ }^{6}$. Já na pesquisa realizada em Ontário, as desordens mentais devido a psicotrópicos, opióides, sedativos e hipnóticos foram as RAM mais prevalentes entre os idosos e equivaliam a $25 \%$ das RAM graves e $33 \%$ das moderadas ${ }^{9}$. O outro estudo canadense revelou que os medicamentos mais associados com as RAM foram antibióticos (15,9\%), anticoagulantes $(14,2 \%)$, agentes antineoplásicos $(9,6 \%)$ e opióides $(7,3 \%)^{10}$.Já nos Estados Unidos, os principais medicamentos relacionados com as RAM entre os idosos foram varfarina, digoxina e insulina. Esses três medicamentos equivaleram a um terço das causas de suspeitas de RAM ${ }^{20}$. Mesmo não tendo sido encontrados neste estudo, observa-se que a maioria dos medicamentos suspeitos de causarem RAM demanda monitoramento da farmacoterapia, demonstrando a importância do monitoramento no uso de medicamentos em pacientes ambulatoriais, para evitar o aparecimento de RAM. Quanto às diferenças nas classes de medicamentos podem ter acontecido devido à condição epidemiológica dos locais estudados.

Muitos trabalhos apontam a idade avançada como fator de risco para o aparecimento de RAM $^{7,9,25}$. Neste trabalho foi observada uma leve predominância de suspeitas de RAM em pacientes com mais de 80 anos. É possível que os idosos que têm idade mais avançada apresentem mais comorbidades e, portanto, utilizem polifarmácia. Além disso, os idosos com idade mais avançada e comorbidades são excluídos dos ensaios clínicos. Assim, a escolha de tratamento para esses pacientes é extrapolada de pacientes mais novos e saudáveis, aumentando o risco de aparecimento de RAM nos pacientes mais idosos ${ }^{26}$.
Alguns trabalhos mostram uma prevalência maior de RAM no sexo feminino, como observado no já citado estudo realizado no Canadá, no qual $60,7 \%$ das RAM ocorreram em mulheres ${ }^{9}$. Pesquisa realizada na Alemanha também mostrou prevalência maior de RAM no sexo feminino, principalmente na faixa etária de 55 a 76 anos. Algumas explicações que justificam essa diferença são os aspectos fisiológicos e hormonais em mulheres que influenciam a farmacocinética e farmacodinâmica dos medicamentos e a constituição diferenciada do organismo feminino que apresenta maior teor de gordura corporal, além de alguns estudos apontarem uma diferença de metabolização hepática dos medicamentos ${ }^{8,27}$. Neste trabalho também a prevalência de RAM foi maior no sexo feminino $(62,5 \%)$. No entanto, deve-se levar em conta também que foram estudados mais prontuários de pacientes do sexo feminino que do masculino.

Todas, as suspeitas de RAM resultaram como "possíveis", de acordo com o algoritmo de Naranjo ${ }^{18}$, devido à falta de informações no prontuário, não sendo possível responder todas as questões propostas pelo instrumento. Nas fichas de atendimento, havia poucos detalhes em relação aos medicamentos que o paciente fazia uso em casa e não havia também informações sobre a prescrição médica na alta do paciente. Também não foi possível saber se o paciente já havia sido reexposto ou não ao medicamento e se a RAM tinha ocorrido pela primeira vez.

À exceção da constipação e diplopia, que não se encaixavam nos critérios de gravidade da ANVISA, as demais suspeitas de RAM foram classificadas como graves.

A seguir, discute-se o uso dos rastreadores testados em pronto socorro adulto:

- O rastreador M1 (Clostridium difficile) não se mostrou um bom rastreador para PSA já que é um exame que nem sempre está disponível e, além disso, seu resultado não é liberado rapidamente. No hospital estudado, esse exame não tem sido realizado também devido à sua baixa sensibilidade. Assim, sua utilização, prospectivamente,em um PSA seria muito difícil.

- Os rastreadores M2 (TTP > 100), M3 (INR > 6) e M6 (vitamina K) não foram encontrados na população estudada. No entanto, a literatura mostra que muitos motivos de atendimento no PSA são devido a anticoagulantes. Dessa forma, são rastreadores importantes na busca de suspeitas de RAM em um PSA.

- O rastreador M4 (glicose<50) foi encontrado em dois prontuários e é importante na de- 
tecção de possíveis RAM em PSA, já que muitos idosos utilizam insulina e/ou hipoglicemiantes orais.

- O marcador M5 (creatinina e ureia) tem grande aplicabilidade, pois vários medicamentos são nefrotóxicos. No entanto, sua presença demanda uma avaliação detalhada do caso, pois a função renal do paciente idoso é facilmente alterada pelo processo de envelhecimento e por outras co-morbidades.

- O rastreador M7 (anti-histamínico) auxilia na identificação de reações de hipersensibilidade, como rash e exantema. Na população de estudo, não foram encontradas essas RAM. Isso pode ter ocorrido porque esse tipo de manifestação, quando é de menor gravidade, em geral, não gera procura por atendimento em PSA, ou porque, nesses casos, o paciente não é mantido em observação. Mesmo assim, como são RAM comuns, é um rastreador importante para ser usado em PS. Além dos anti-histamínicos, poderiam ser adicionados "rash" e "prurido" como rastreadores para identificar RAM de hipersenbilidade.

- Os rastreadores M8 (flumazenil) e M9 (naloxona) não apresentam grande aplicabilidade como rastreadores no PSA. Como são antagonistas de benzodiazepínicos e opióides, são mais importantes na internação e para identificação de intoxicação.

- O rastreador M10 (anti-eméticos), neste trabalho, foi considerado quando o anti-emético era administrado no início do atendimento do paciente no PSA, podendo, assim, indicar relação com uma RAM de um medicamento que o paciente fazia uso em casa. Náuseas e vômitos são sintomas comuns em várias doenças e são RAM de vários medicamentos. Dessa forma, é um rastreador que exige atenção na hora da análise.

- O rastreador M11 (hipotensão/supersedação) é essencial para uso em PS, já que anti-hipertensivos são medicamentos comumente utilizados por idosos. Neste trabalho, identificou duas suspeitas de RAM.

- O rastreador M12 (interrupção abrupta do medicamento) é de difícil utilização em estudo retrospectivo, porque não é possível saber se a interrupção foi realmente abrupta, já que não se sabe como o paciente fazia uso do medicamento. Além disso, sem informações sobre a receita de alta de paciente, não é possível saber se houve continuidade ou não do medicamento após o atendimento no PS. Entretanto, trata-se de um rastreador aplicável para estudos prospectivos.

- O rastreador M13 (outros): na busca de ativa de RAM foram encontrados mais dois importantes rastreadores para o PSA: constipação e nível sérico de sódio. O sódio é um importante rastreador de RAM causada por diuréticos.

Por se tratar de um estudo retrospectivo, este trabalho apresentou como limitação a falta de informações detalhadas nas evoluções clínicas e da equipe multiprofissional em relação às suspeitas de RAM e medicamentos que o paciente fazia uso antes da admissão no PSA, além da prescrição originada na visita ao PS. A posologia dos medicamentos utilizados no domicílio geralmente estava incompleta no prontuário e não foi possível confirmar com o paciente essas informações. A falta de informações nos prontuários limitou a avaliação das RAM. A falta de prontuários e prescrições eletrônicas também foi um grande limitante do trabalho retrospectivo. Também não foi pesquisada a performance dos rastreadores para uso em PSA, já que não havia um padrão das RAM ocorridas no serviço estudado para o cálculo de sensibilidade e especificidade. Entretanto, trabalho realizado no Canadá, pesquisou esses parâmetros e observou uma baixa sensibilidade, de 2,6 a $15,8 \%$, dependendo do rastreador, mas uma alta especificidade. Os autores sugerem que rastreadores mais sensíveis podem ser desenvolvidos usando-se métodos de decisão clínica, nos quais o julgamento clínico é inicialmente utilizado para definir candidatos a preditores ${ }^{28}$.

Mesmo com essas limitações, pode-se concluir que é possível realizar busca de reações adversas a medicamentos em pronto socorro utilizando-se rastreadores do IHI. Vários deles mostraram-se aplicáveis, com algumas sugestões. Sugerem-se trabalhos prospectivos e com maior número de pessoas para consolidar o quadro de rastreadores do IHI úteis para cada serviço e para calcular a performance dos rastreadores na identificação de suspeitas de RAM em idosos que procuram atendimento em PS. Assim, este trabalho permitiu obter um perfil de suspeitas de RAM que ocorrem fora do ambiente hospitalar, sinalizando para medicamentos que podem causar reações mais comuns nessa população e que, portanto, necessitam maior atenção para aumentar a segurança do paciente em relação ao uso de medicamentos. 


\section{Colaboradores}

KL Nagai trabalhou na concepção da pesquisa, na coleta e interpretação dos dados e na redação do artigo. PSK Takahashi trabalhou na concepção da pesquisa, na interpretação dos dados, na redação do artigo e em sua revisão crítica. LMO Pinto trabalhou na revisão crítica. NS Romano -Lieber trabalhou na concepção da pesquisa, na redação do artigo e em sua revisão crítica.

\section{Referências}

1. Organización Mundial de la Salud (OMS). La farmacovigilancia: garantía de seguridad en el uso de los medicamentos. Perspect políticas la OMS sobre Medicam 2004; $1-6$.

2. Vincent C, Neale G, Woloshynowych M. Adverse events in British hospitals: preliminary retrospective record review. BMJ 2001; 322(7285):517-519.

3. Bouvy JC, De Bruin ML, Koopmanschap MA. Epidemiology of adverse drug reactions in Europe: a review of recent observational studies. Drug Saf 2015; 38(5):437-453.

4. Miguel L, Azevedo LF, Araújo M PA. Frequency of adverse drug reactions in hospitalized patients: a systematic review and meta-analysis. Pharmacoepidemiol Drug Saf 2012; 21(11):1139-1154.

5. Lazarou J, Pomeranz BH, Corey PN. Incidence of adverse drug reactions in hospitalized patients: a meta-analysis of prospective studies. JAMA 1998; 279(15):1200.

6. Kongkaew C, Noyce PR, Ashcroft DM. Hospital admissions associated with adverse drug reactions: a systematic review of prospective observational studies. Ann Pharmacother 2008; 42(7):1017-1025.

7. Beijer HJM, De Blaey CJ. Hospitalisations caused by adverse drug reactions (ADR): A meta-analysis of observational studies. Pharm World Sci 2002; 24(April 2000):46-54.

8. Varallo FR, Matroianni PC. Farmacovigilância da teoria à prática. São Paulo. Unesp; 2013.

9. Wu C, Bell CM, Wodchis WP. Incidence and Economic Burden of Adverse Drug Reactions among Elderly Patients in Ontario Emergency Departments. Drug Saf 2012; 35(9):769-781.

10. Bayoumi I, Dolovich L, Hutchison B, Holbrook A. Medication-related emergency department visits and hospitalizations among older adults. Can Fam Physician 2014; 60(4):e217-222.

11. Domingues PHF, Galvão TF, Andrade KRC, Sá PTT, Silva MT, Pereira MG. Prevalence of self-medication in the adult population of Brazil: a systematic review. Rev Saude Publica 2015; 49:36.

12. Rozich JD, Haraden CR, Resar RK. Adverse drug event trigger tool: a practical methodology for measuring medication related harm. Qual Saf Health Care 2003; 12(3):194-200.

13. Griffin F, Resar R. IHI Global Trigger Tool for measuring adverse events. IHI Innov Ser white Pap. 2009; 1-44. [acessado 2015 Fev 20]. Disponível em: http://www.ihi. org/resources/Pages/IHIWhitePapers/IHIGlobalTriggerToolWhitePaper.aspx

14. World Health Organization (WHO). The Uppsala Monitoring Centre. The Importance of Pharmacovigilance. Safety monitoring of medicinal products. 2002; 1-38 [acessado 2015 Mar 10]. Disponível em: http:// apps.who.int/medicinedocs/pdf/s4893e/s4893e.pdf

15. World Health Organization (WHO). Guideline for ATC classification and DDD assignment 2016. Oslo: WHO Collaborating Centre for Drug Statistics Methodology; 2015. [acessado 2016 Jan 10]. Disponível em: http:// www.whocc.no/filearchive/publications/2016_guidelines_web.pdf 
16. World Health Organization (WHO). The Uppsala Monitoring Centre. The WHO Adverse Reaction Terminology-WHO-ART. 2014. [acessado 2015 Fev 20]. Disponível em: http://www.umc-products.com/graphics/28010.pdf

17. Brasil. Agência Nacional de Vigilância Sanitária (Anvisa). Diretrizes para o Gerenciamento do Risco em Farmacovigilância 2008; 1-14. [acessado 2015 Out 15]. Disponível em: http://portal.anvisa.gov.br/wps/wcm/ connect/4140a10047cd94dc9845fed498087ae1/Diretrizes_para_o_GRFV.pdf?MOD=AJPERES

18. Naranjo C, Busto U, Sellers EM, Sandor P, Ruiz I, Roberts EA, Janecek E, Domecq C, Greenblatt DJ. A method for estimating the probability of adverse drug reactions. Clin Pharmacol Ther 1981; 30(2):239-245.

19. Pedrós C, Formiga F, Corbella X, Arnau JM. Adverse drug reactions leading to urgent hospital admission in an elderly population: Prevalence and main features. Eur J Clin Pharmacol 2016; 72(2):219-226.

20. Budnitz DS, Pollock DA, Weidenbach KN, Mendelsohn AB, Schroeder TJ, Annest JL. National surveillance of emergency department visits for outpatient adverse drug events. JAMA 2006; 296(15):1858-1866.

21. Andreazza RS, Silveira de Castro M, Sippel Köche P, Heineck I. Causes of drug-related problems in the emergency room of a hospital in southern Brazil. Gac Sanit 2011; 25(6):501-506.

22. Brasil. Lei no 10.741, de 1 de outubro de 2003. Dispõe sobre o Estatuto do Idoso e dá outras providências. Diário Oficial da União 2003; 3 out.

23. Alhawassi TM, Krass I, Bajorek B V, Pont LG. A systematic review of the prevalence and risk factors for adverse drug reactions in the elderly in the acute care setting. Clin Interv Aging 2014; 9(July 2016):2079-2086.

24. Brasil. Ministério da Saúde (MS). DataSUS Informações de Saúde (TABNET). [acessado 2015 maio 1]. Disponível em: http://www2.datasus.gov.br/DATASUS/ index. php?area $=0207$ \&item $=$

25. Zopf Y, Rabe C, Neubert A, Hahn EG, Dormann H. Risk factors associated with adverse drug reactions following hospital admission: a prospective analysis of 907 patients in two German university hospitals. Drug Saf 2008; 31(9):789-798.

26. Hubbard RE, O'Mahony MS, Woodhouse KW. Medication prescribing in frail older people. Eur J Clin Pharmacol 2013; 69(3):319-326.

27. Zopf Y, Rabe C, Neubert A, Gaßmann KG, Rascher W, Hahn EG, Brune K, Dormann H. Women encounter ADRs more often than do men. Eur J Clin Pharmacol 2008; 64(10):999-1004.

28. Karpov A, Parcero C, Mok C, Panditha C, Yu E, Dempster L, Hohl CM. Performance of Trigger Tools in Identifying Adverse Drug Events in Emergency Department Patients: A Validation Study. Br J Clin Pharmacol 2016; 82(4):1048-1057.

Artigo apresentado em 27/09/2016

Aprovado em 28/11/2016

Versão final apresentada em 30/11/2016 